\title{
Efeitos da extração hidroetanolica e sequencial sobre o potencial tóxico dos extratos de diferentes partes da Parkia platycephala do Cerrado tocantinense
}

\author{
Effects of hydroethanolic and sequential extraction on the toxic potential of extracts from different \\ parts of Parkia platycephala from the Cerrado of Tocantins
}

Efectos de la extracción hidroetanólica y secuencial sobre el potencial tóxico de extractos de diferentes partes de Parkia platycephala del Cerrado de Tocantins

\author{
Rachel de Moura Nunes Fernandes \\ ORCID: https://orcid.org/0000-0002-4524-8961 \\ Universidade Federal do Tocantins, Brasil \\ E-mail: rachelnunes@uft.edu.br \\ Tais Souza dos Santos Dias \\ ORCID: https://orcid.org/0000-0001-6296-2270 \\ Universidade Federal do Tocantins, Brasil \\ E-mail: tais.dias@uft.edu.br \\ Marcelo Henrique Toscano da Silva \\ ORCID: https://orcid.org/0000-0001-6917-0006 \\ Universidade Federal do Tocantins, Brasil \\ E-mail: marc-soul@hotmail.com \\ Elisandra Scapin \\ ORCID: https://orcid.org/0000-0001-7506-308X \\ Universidade Federal do Tocantins, Brasil \\ E-mail: scapin@uft.edu.br
}

\begin{abstract}
Resumo
Comumente percebe-se o uso de plantas como medicamentos fitoterápicos, entretanto, a linha tênue entre o tratamento e a intoxicação é uma realidade. Este estudo avaliou a toxicidade dos extratos da folha, casca, flor e semente da Parkia platycephala através do crescimento radicular da Allium cepa. Foram realizadas duas metodologias de extração, uma polar e uma sequencial, ambas usando aparato Soxhlet, obtendo-se extratos brutos (FEB, CEB, FREB, SEB), metanolicos (FEM, CEB, FREM, SEM) e etanolicos (FEE, CEE, FREE, SEE) da folha, casca, flor e semente, respectivamente. O potencial tóxico de cada extrato foi avaliado em três concentrações $(50,250,750 \mu \mathrm{g} / \mathrm{ml})$, tendo a água mineral como controle negativo. Observou-se que das 36 amostras analisadas, $25 \%$ estimularam o crescimento radicular da A. cepa nas condições avaliadas e 39\% mantiveram as características de crescimento do controle negativo, indicando não toxicidade desses extratos. Os melhores índices de crescimento radicular foram obtidos na casca $(2,7) \mathrm{e}$ flor $(2,0)$ pela metodologia sequencial. O extrato bruto da casca (CEB) não apresentou toxicidade nas concentrações avaliadas. Os extratos da semente, da extração sequencial, apresentaram toxicidade a partir da concentração $250 \mu \mathrm{g} / \mathrm{ml}$. $\mathrm{Na}$ concentração de $750 \mu \mathrm{g} / \mathrm{ml}$, apenas um extrato não apresentou toxicidade. Diante dos resultados apresentados, acredita-se no potencial uso terapêutico da espécie $P$. platycephala nas concentrações inferiores a $250 \mu \mathrm{g} / \mathrm{ml}$, no entanto é necessário estudos mais detalhados para confirmação da toxicidade dos extratos da espécie.
\end{abstract}

Palavras-chave: Intoxicação; Alelopatia; Fava de bolota; Allium cepa; Brasil.

\begin{abstract}
Commonly, the use of plants as herbal medicines is perceived, however, the fine line between treatment and intoxication is a reality. This study evaluated the toxicity of extracts from the leaf, bark, flower and seed of Parkia platycephala through root growth of Allium cepa. Two extraction methodologies were carried out, one polar and one sequential, both using Soxhlet apparatus, obtaining crude extracts (FEB, CEB, FREB, SEB), methanolic (FEM, CEB, FREM, SEM) and ethanolic (FEE, CEE, FREE, SEE) of leaf, bark, flower and seed, respectively. The toxic potential of each extract was evaluated at three concentrations $(50,250,750 \mu \mathrm{g} / \mathrm{ml})$, with mineral water as a negative control. It was observed that of the 36 samples analyzed, 25\% stimulated the root growth of $A$. cepa under the conditions evaluated and $39 \%$ maintained the growth characteristics of the negative control, indicating non-toxicity of these extracts. The best rates of root growth were obtained in the bark (2.7) and flower (2.0) by the sequential methodology. The crude bark extract (CEB) did not show toxicity at the concentrations evaluated. Seed extracts from sequential extraction showed toxicity from the concentration of $250 \mu \mathrm{g} / \mathrm{ml}$. At a concentration of $750 \mu \mathrm{g} / \mathrm{ml}$, only one extract showed no toxicity. In view of
\end{abstract}


the results presented, it is believed in the potential therapeutic use of the species $P$. platycephala at concentrations below $250 \mu \mathrm{g} / \mathrm{ml}$, however more detailed studies are needed to confirm the toxicity of the extracts of the species.

Keywords: Intoxication; Allelopathy; Fava de bolota; Allium cepa; Brazil.

\begin{abstract}
Resumen
Comúnmente se percibe el uso de las plantas como remedios herbales, sin embargo, la delgada línea entre el tratamiento y la intoxicación es una realidad. Este estudio evaluó la toxicidad de extractos de hoja, corteza, flor y semilla de Parkia platycephala a través del crecimiento de raíces de Allium cepa. Se llevaron a cabo dos metodologías de extracción, una polar y otra secuencial, ambas con aparato Soxhlet, obteniendo extractos crudos (FEB, CEB, FREB, SEB), metanólicos (FEM, CEB, FREM, SEM) y etanólicos (FEE, CEE, FREE, SEE) de hoja, corteza, flor y semilla, respectivamente. El potencial tóxico de cada extracto se evaluó a tres concentraciones $(50,250,750 \mu \mathrm{g} / \mathrm{ml})$, con agua mineral como control negativo. Se observó que de las 36 muestras analizadas, el $25 \%$ estimuló el crecimiento radicular de A. cepa en las condiciones evaluadas y el $39 \%$ mantuvo las características de crecimiento del control negativo, indicando la no toxicidad de estos extractos. Las mejores tasas de crecimiento de raíces se obtuvieron en corteza $(2,7)$ y flor $(2,0)$ mediante la metodología secuencial. El extracto crudo de corteza (CEB) no presentó toxicidad a las concentraciones evaluadas. Los extractos de semillas de la extracción secuencial mostraron toxicidad a partir de la concentración de 250 $\mu \mathrm{g} / \mathrm{ml}$. A una concentración de $750 \mu \mathrm{g} / \mathrm{ml}$, solo un extracto no mostró toxicidad. A la vista de los resultados presentados, se cree en el potencial uso terapéutico de la especie P. platycephala en concentraciones inferiores a $250 \mu \mathrm{g} / \mathrm{ml}$, sin embargo, se necesitan estudios más detallados para confirmar la toxicidad de los extractos de la especie.
\end{abstract}

Palabras clave: Intoxicación; Alelopatía; Fava de bolota; Allium cepa; Brasil.

\title{
1. Introdução
}

Há uma preocupação crescente na pesquisa de plantas medicinais devido à descoberta de novos compostos naturais com potencial farmacológico, entretanto esse potencial pode apresentar risco toxicológico, ocasionando intoxicação nos organismos expostos a tais componentes (Tuttolomondo et al, 2014).

O uso da Allium cepa (A. серa) como bioindicador de toxicidade em extratos de plantas, é uma alternativa viável em substituição a testes em animais (Viega et al., 2019), além de possuir elevada sensibilidade e boa correlação com outros sistemastestes, tal como ensaios em mamíferos (Freires et al., 2017). Através deste teste é possível obter significativos efeitos macromorfológicos em um curto espaço de tempo (Bonciu et al., 2018; Bragantini et al., 2007; Brigido et al., 2016). Tais efeitos, são devido a influência de um corpo sobre o outro, que pode ou não favorecer o desenvolvimento do organismo alvo. Esse fenômeno denominado alelopatia, é uma característica fitotóxica observada em vários biomas (Inderjit et al., 2011).

As plantas do bioma Cerrado por serem capazes de sobreviver em condições edafoclimáticas extremas e intensa competição por nutrientes, têm a produção de compostos bioativos estimulada, o que influencia nas diferentes interações nesse ambiente (Mecina et al., 2014). Todas essas características tornam o Cerrado uma fonte promissora de novos compostos aleloquímicos.

A espécie Parkia platycephala (P. platycephala), endêmica do Cerrado brasileiro, apresenta potencial anti-helmíntico, gastroprotetor, antinociceptivo e anti-inflamatório, devido à presença de alguns metabólitos ativos (Bari et al., 2016; Figueiredo et al., 2021; Silva et al., 2019). Além desses potenciais farmacológicos, a $P$. platycephala apresenta a capacidade de restringir o desenvolvimento de outras espécies abaixo de sua copa, muito embora o risco toxicológico dos extratos desta espécie, não tenham sido antes averiguados.

Diante disso, esse estudo avaliou o potencial tóxico dos extratos da folha, casca, flor e semente da $P$. platycephala pelo método com A. cepa, com fins de nortear o uso terapêutico seguro dessa espécie.

\section{Metodologia}

\subsection{Coleta e preparo}

A coleta da folha, casca, flor e da semente da Parkia platycephala e ocorreu dentro da Universidade Federal do

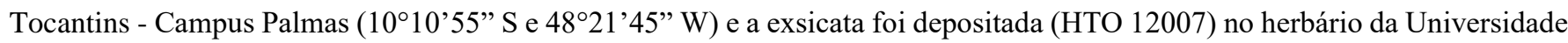


Federal do Tocantins, no município de Porto Nacional. Realizou-se também o cadastro junto ao SISGEN sob número A06B860. As etapas de secagem (estufa, $60^{\circ} \mathrm{C} / 48 \mathrm{~h}$ ) e pulverização (moinho de facas tipo Willey) foram utilizadas no preparo das amostras, que logo em seguida foram armazenadas em frascos de vidros fechados e em ambiente livre de luz (Anvisa, 2011).

\subsection{Extração}

Duas metodologias extrativas foram analisadas, ambas à quente em sistema fechado por meio do equipamento Soxhlet conforme recomendado por Oliveira et al. (2016) e Soares et al (2017), com modificações. Os extratos brutos foram obtidos por extração polar, utilizando $200 \mathrm{~mL}$ da solução hidroetanólica (70\%) e $5 \mathrm{~g}$ da amostra pulverizada, durante $5 \mathrm{~h}$ de refluxo. Os extratos metanolicos e etanolicos foram obtidos por extração sequencial, com prévio desengorduramento com hexano. Após o desengorduramento, a amostra foi deixada em repouso por $12 \mathrm{~h}$ para secagem e em seguida realizada a extração metanólica $(200 \mathrm{~mL} / 5 \mathrm{~g})$ por $5 \mathrm{~h}$. Novo período de descanso (12h) foi aguardado para secagem e assim realizada a última extração com a solução hidroetanolica (70\%), nas mesmas condições. Assim na extração polar, obtiveram-se os extratos brutos da folha (FEB), da casca (CEB), da flor (FREB) e da semente (SEB). Na extração sequencial, os extratos metanolicos da folha (FEM), da casca (CEM), da flor (FREM) e da semente (SEM) e os extratos etanolicos da folha (FEE), da casca (CEE), da flor (FREE) e da semente (SEE).

\subsection{Determinação da Toxicidade}

Os experimentos de toxicidade utilizando espécimes de Allium cepa (A. серa) foram conduzidos conforme recomendado por Martins et al (2016) com alterações. O delineamento experimental incluiu um delineamento fatorial $3 \times 3$ (três extratos e três concentrações) para cada parte da planta, além de um tratamento controle (sem extrato). Inicialmente avaliou-se a capacidade de germinação das cebolas. As mesmas foram preparadas retirando-se a casca e quaisquer sinais de raiz. Em seguida colocou-se o fundo da cebola em copos descartáveis $(50 \mathrm{~mL})$ contendo água mineral como controle negativo, em temperatura de $25^{\circ} \mathrm{C}$, atentando-se para que o fundo ficasse submerso no líquido. Após $48 \mathrm{~h}$ as cebolas foram analisadas e apenas as que tiveram crescimento de raiz e não apresentaram sinais de apodrecimento, foram separadas para a etapa seguinte. As cebolas que estavam aptas para a próxima etapa foram preparadas, retirando-se qualquer raiz presente. Foram preparadas soluções de concentrações variadas $(50,250$ e $750 \mu \mathrm{g} / \mathrm{ml})$. Os extratos foram colocados em triplicata em copos descartáveis $(50 \mathrm{~mL})$ e as cebolas foram submersas nos mesmos pelo período de $120 \mathrm{~h}$ a $25^{\circ} \mathrm{C}$. Após o período de germinação as três raízes principais de cada bulbo (para um total de 9 raízes por ensaio) foram medidas com um paquímetro digital em mm. Foi utilizada análise de variância (ANOVA) para comparar os valores médios obtidos nas análises e os valores $\mathrm{p}<0,05$ foram considerados estatisticamente significativos pelo teste de Tukey. O Índice de Crescimento Relativo (RGI) foi calculado de acordo com Young et al. (2012). $\mathrm{Na}$ análise de RGI, extratos com valores abaixo de 0,8 foram classificados como inibidores do crescimento; valores entre $0,8 \mathrm{e}$ 1,2 não indicaram efeito significativo e valores a partir de 1,2 evidenciaram estímulo do crescimento radicular.

\section{Resultados e Discussão}

O crescimento radicular de A. cepa em resposta ao contato com os extratos da folha da P. platycephala está disposto na Figura 1. 
Figura 1. Crescimento da raiz da A. cepa em função da concentração dos extratos da folha da $P$. platycephala ((FEB - extrato bruto folha; FEM - extrato metanolico folha; FEE - extrato etanolico folha). Letras maiúsculas iguais indicam semelhança significativa na mesma concentração e letras minúsculas em concentrações diferentes ( $\mathrm{p}<0.05$, seguido de teste Tukey).

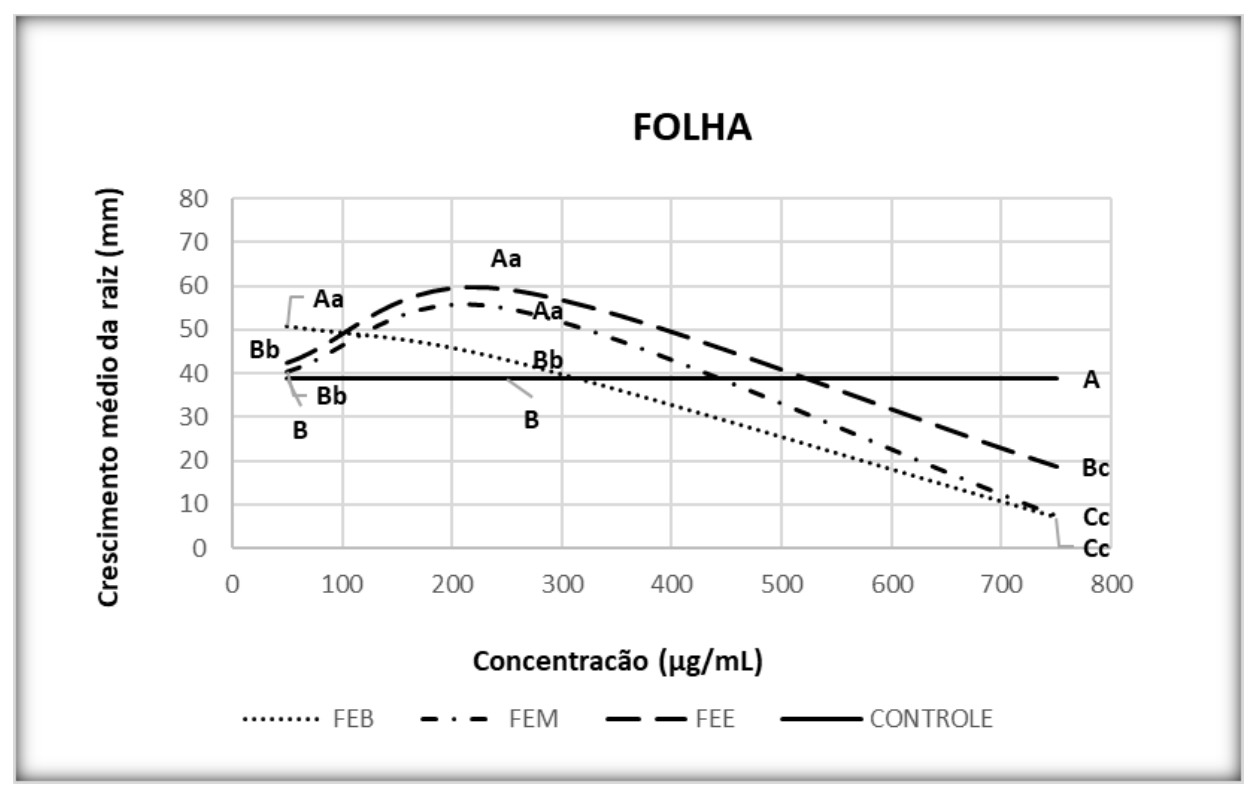

Fonte: Autores (2022).

Observou-se nos extratos da folha que as duas metodologias utilizadas apresentaram crescimento radicular da $A$. cepa, embora em concentrações diferentes. Em ambos os extratos obtidos por extração sequencial (FEM, FEE), na concentração de $250 \mu \mathrm{g} / \mathrm{ml}$, foram detectados os maiores índices de crescimento radicular. Houve diferença significativa nos valores obtidos, tanto em relação a variação de concentração de cada extrato, como em relação as metodologias utilizadas.

$\mathrm{Na}$ Figura 2, estão representados os crescimentos radiculares de A. cepa em resposta ao contato com os extratos da casca da P. platycephala. 
Figura 2. Crescimento da raiz da $A$. cepa em função da concentração dos extratos da casca da $P$. platycephala (CEB - extrato bruto casca; CEM - extrato metanolico casca; CEE - extrato etanolico casca). Letras maiúsculas iguais indicam semelhança significativa na mesma concentração e letras minúsculas em concentrações diferentes ( $\mathrm{p}<0.05$, seguido de teste Tukey).

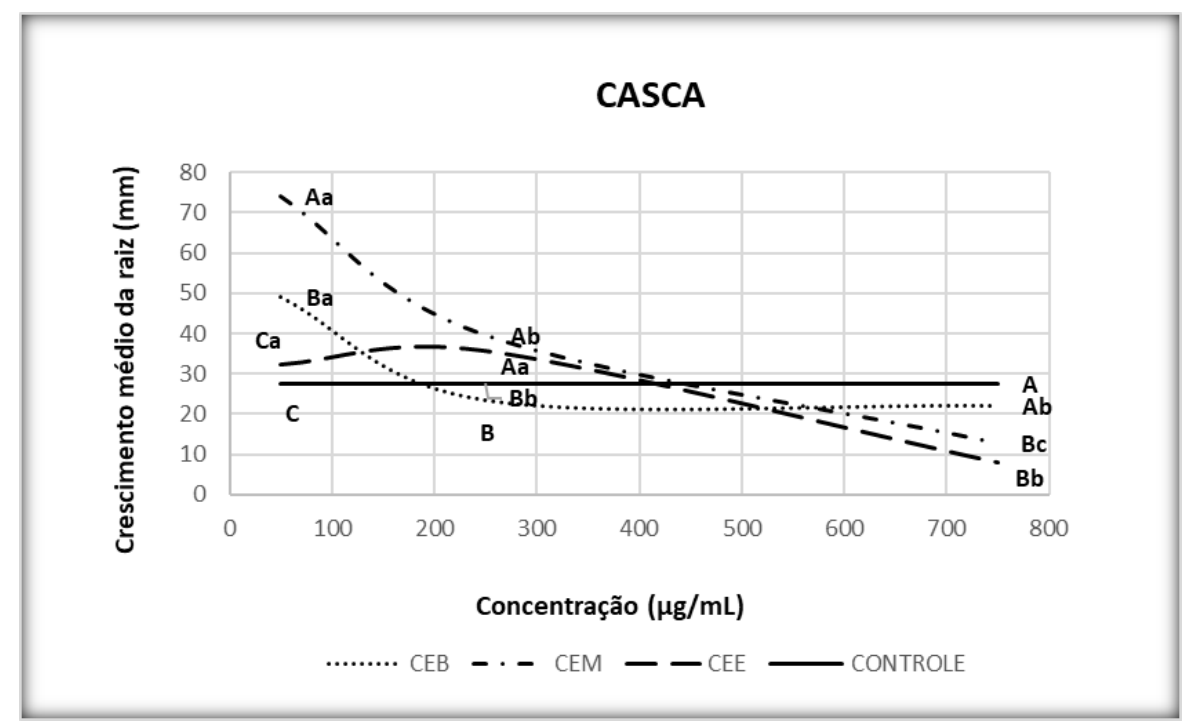

Fonte: Autores (2022).

$\mathrm{Na}$ casca, cada extrato apresentou uma característica peculiar. Na extração sequencial, entre as concentrações de 50$250 \mu \mathrm{g} / \mathrm{ml}$, houve tanto diminuição (CEM) como estabilidade (CEE) dos índices de crescimento radicular. Já na extração polar, ressalta-se que o CEB, a partir da concentração de $250 \mu \mathrm{g} / \mathrm{ml}$, manteve as condições de crescimento radicular equivalentes as do controle negativo, ou seja, não apresentou dose dependência.

Na Figura 3, estão representados os crescimentos radiculares de A. cepa em resposta ao contato com os extratos da flor da P. platycephala.

Figura 3. Crescimento da raiz da A. cepa em função da concentração dos extratos da flor da $P$. platycephala (FREB - extrato bruto flor; FREM - extrato metanolico flor; FREE - extrato etanolico flor). Letras maiúsculas iguais indicam semelhança significativa na mesma concentração e letras minúsculas em concentrações diferentes ( $\mathrm{p}<0.05$, seguido de teste Tukey).

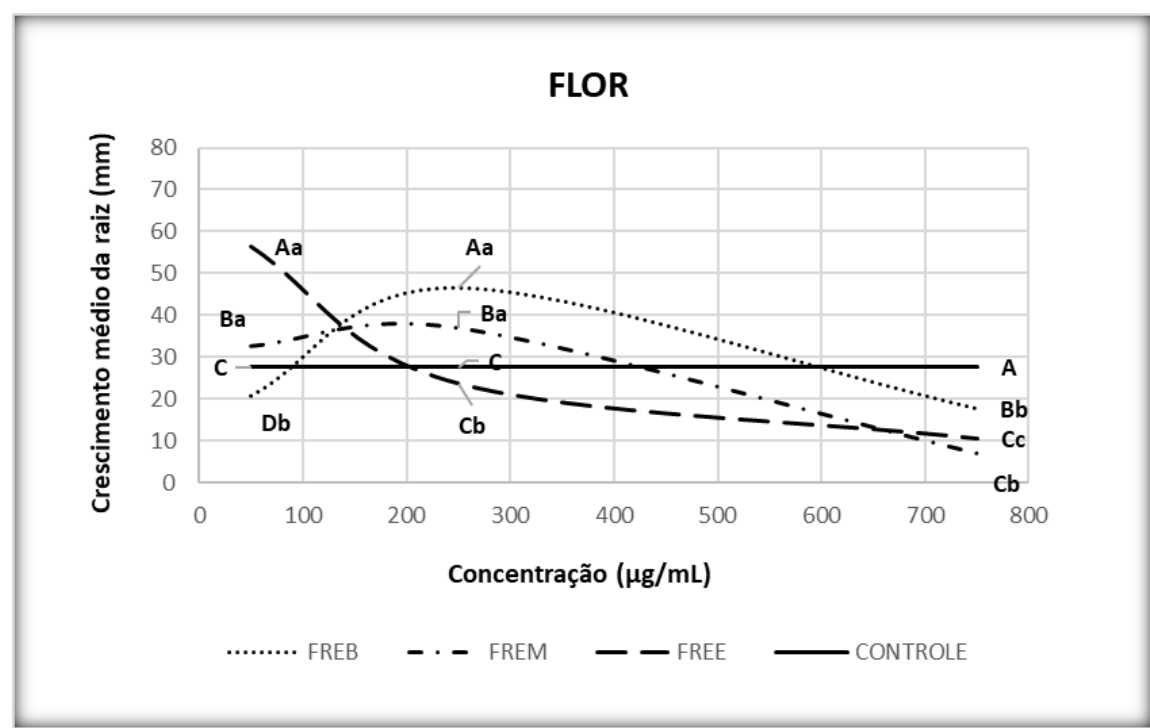

Fonte: Autores (2022). 
Os extratos da flor apresentaram resultados de crescimento radiculares positivos para ambas as metodologias, destacando-se o FREE em $50 \mu \mathrm{g} / \mathrm{ml}$ e o FREB em $250 \mu \mathrm{g} / \mathrm{ml}$. Observou-se, entretanto, que tais extratos apresentam características opostas em relação à dose dependência, entre as concentrações de 50 a $250 \mu \mathrm{g} / \mathrm{ml}$, enquanto o FREE apresentou diminuição no crescimento radicular, o FREB favoreceu o crescimento.

Na Figura 4, estão representados os crescimentos radiculares de A. cepa em resposta ao contato com os extratos da semente da $P$. platycephala.

Figura 4. Crescimento da raiz da $A$. cepa em função da concentração dos extratos da semente da $P$. platycephala (SEB - extrato bruto semente; SEM - extrato metanolico semente; SEE - extrato etanolico semente). Letras maiúsculas iguais indicam semelhança significativa na mesma concentração e letras minúsculas em concentrações diferentes $(p<0.05$, seguido de teste Tukey).

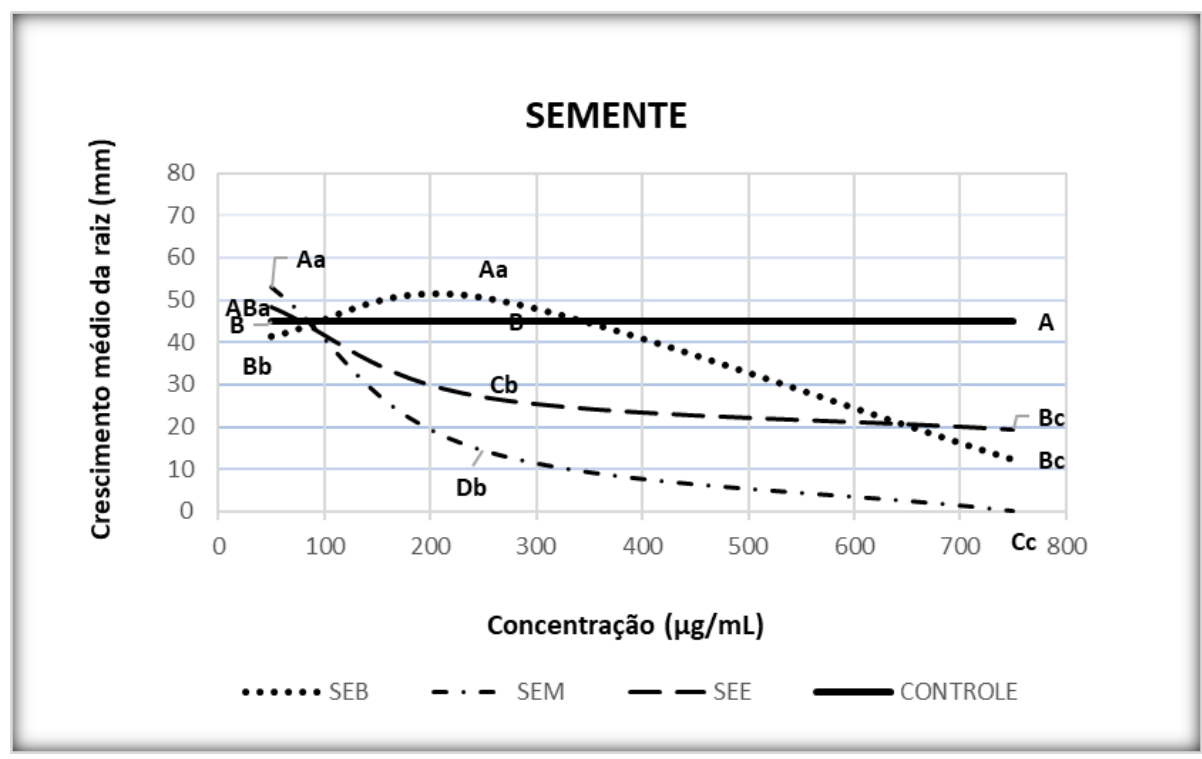

Fonte: Autores (2022).

Analisando os extratos da semente, houve influência das metodologias de extração no crescimento radicular até a concentração de $250 \mu \mathrm{g} / \mathrm{ml}$. O extrato obtido por extração polar (SEB) respondeu positivamente ao crescimento, enquanto ambos os extratos obtidos por extração sequencial (SEM, SEE) apresentaram resultados negativos de crescimento em relação ao controle negativo.

Na concentração de $750 \mu \mathrm{g} / \mathrm{ml}$, apenas o CEB mostrou o crescimento equivalente ao controle, os demais extratos responderam negativamente ao crescimento radicular.

Diante disso, para a análise de toxicidade dos extratos da folha, casca, flor e semente da P. platycephala, fez-se uso do RGI (Tabela 1). 
Tabela 1. Índice de crescimento relativo (RGI) observado em extratos de diferentes partes da P. platycephala. (FEB - extrato bruto folha; FEM - extrato metanolico folha; FEE - extrato etanolico folha; CEB - extrato bruto casca; CEM - extrato metanolico casca; CEE- extrato etanolico casca; FREB - extrato bruto flor; FREM - extato metanolico flor; FREE - extrato etanolico flor; SEB - extrato bruto semente; SEM - extrato metanolico semente; SEE - extrato etanlico semente).

\begin{tabular}{c|c|c|c}
\hline \multirow{2}{*}{ EXTRATO } & \multicolumn{3}{|c}{ RGI } \\
\cline { 2 - 4 } & $\mathbf{5 0}(\boldsymbol{\mu g} / \mathbf{m l})$ & $\mathbf{2 5 0}(\boldsymbol{\mu g} / \mathbf{m l})$ & $\mathbf{7 5 0}(\boldsymbol{\mu g} / \mathbf{m l})$ \\
\hline FEB & $1,3[\mathrm{E}]$ & $1,1[\mathrm{NA}]$ & $0,2[\mathrm{I}]$ \\
\hline FEM & $1,0[\mathrm{NA}]$ & $1,4[\mathrm{E}]$ & $0,2[\mathrm{I}]$ \\
\hline FEE & $1,1[\mathrm{NA}]$ & $1,5[\mathrm{E}]$ & $0,5[\mathrm{I}]$ \\
\hline CEB & $1,8[\mathrm{E}]$ & $0,9[\mathrm{NA}]$ & $0,8[\mathrm{NA}]$ \\
\hline CEM & $2,7[\mathrm{E}]$ & $1,4[\mathrm{E}]$ & $0,5[\mathrm{I}]$ \\
\hline CEE & $1,2[\mathrm{E}]$ & $1,3[\mathrm{E}]$ & $0,3[\mathrm{I}]$ \\
\hline FREB & $0,8[\mathrm{NA}]$ & $1,7[\mathrm{E}]$ & $0,6[\mathrm{I}]$ \\
\hline FREM & $1,2[\mathrm{E}]$ & $1,3[\mathrm{E}]$ & $0,2[\mathrm{I}]$ \\
\hline FREE & $2,0[\mathrm{E}]$ & $0,9[\mathrm{NA}]$ & $0,4[\mathrm{I}]$ \\
\hline SEB & $0,9[\mathrm{NA}]$ & $1,1[\mathrm{NA}]$ & $0,3[\mathrm{I}]$ \\
\hline SEM & $1,2[\mathrm{E}]$ & $0,3[\mathrm{I}]$ & $0,0[\mathrm{I}]$ \\
\hline SEE & $1,1[\mathrm{NA}]$ & $0,6[\mathrm{I}]$ & $0,4[\mathrm{I}]$ \\
\hline
\end{tabular}

[E] - estimulo; [NA] - nenhuma alteração; [I] - inibição. Fonte: Autores (2022).

Observou-se que das 36 amostras analisadas, 25\% estimularam o crescimento radicular da $A$. cepa nas condições avaliadas e $39 \%$ mantiveram as características de crescimento do controle negativo.

Os extratos FEM e FEE, na concentração de $250 \mu \mathrm{g} / \mathrm{ml}$, estimularam o crescimento radicular da $A$. cepa. O mesmo efeito foi observado para o FEB, entretanto na concentração $50 \mu \mathrm{g} / \mathrm{ml}$. Isso sugere que entre as concentrações de $250-750 \mu \mathrm{g} / \mathrm{ml}$, os extratos da folha da $P$. platycephala começam a apresentar algum grau de toxicidade, resultando na inibição do crescimento na última faixa de concentração analisada.

Em relação à casca, os extratos obtidos por extração sequencial estimularam o crescimento radicular da $A$. cepa, destacando-se o CEM, que na concentração de $50 \mu \mathrm{g} / \mathrm{ml}$, obteve RGI=2,7, o maior índice dentre todos os extratos da planta. Notou-se também, que o extrato obtido pela extração polar (CEB) não apresentou toxicidade nas concentrações testadas.

Já para a flor da $P$. platycephala, nas concentrações abaixo de $250 \mu \mathrm{g} / \mathrm{ml}$, os extratos obtidos por extração sequencial (FREM, FREE) não apresentaram toxicidade, destacando-se o FREE com RGI=2,0 na concentração de $50 \mu \mathrm{g} / \mathrm{ml}$.

A extração sequencial da semente, gerou resultados diferenciados, pois o SEM e o SEE, foram os únicos extratos que apresentaram toxicidade a partir da concentração de $250 \mu \mathrm{g} / \mathrm{ml}$.

$\mathrm{Na}$ literatura não foram encontrados estudos anteriores sobre a toxicidade da espécie $P$. platycephala ou do gênero Parkia, pelo método de crescimento radicular da A. cepa.

Há relatos do efeito inibidor da Parkia biglobosa (P. biglobosa) na germinação de sementes de Striga gesnerioides (Itta et al., 2014; Lado et al., 2018), bem como na germinação da Striga hermonthica (Magani et al., 2010; Mamudu et al., 2019). Estudos mais recentes com a folha e semente da $P$. platycephala, detectaram toxicidade moderada no extrato etanolico da semente e baixa toxicidade nos extratos metanolicos da folha e semente, pelo método com Artemia salina (Fernandes et al., 2021). Também foi detectado elevado potencial tóxico da P. biglobosa em Clarias gariepinus (Bawa-Allah e kinnuoye, 2019).

Em relação à toxicidade de plantas do Cerrado, alguns estudos analisaram a toxicidade de espécies desse bioma através da atividade alelopática de seus extratos. Almeida et al. (2021) utilizando A. cepa, avaliaram a toxicidade do extrato aquoso da 
folha da Vernonanthura polyanthes (assa-peixe) e observaram efeitos tóxicos da espécie em concentrações superiores a 20 $\mathrm{mg} / \mathrm{ml}$. Chiavegatto et al. (2017) relataram que os extratos metanolicos do fruto da Solanum lycocarpum (lobeira) têm um efeito inibitório sobre o crescimento das raízes tanto da A. cepa quanto da Lactuca sativa.

Extratos hidroetanolicos da casca de outra espécie do Cerrado, a Astronium fraxinifolium (Gonçalo-alves), também apresentou toxicidade, nos testes com A. cepa (Zafred et al., 2016). Segundo Panontin et al. (2021), foi confirmada a toxicidade dos extratos hidroetanolicos da casca e folha da espécie Hancornia speciosa (mangabeira), tanto pelo crescimento radicular como pelas células mitóticas da A. cepa em concentrações de $500 \mu \mathrm{g} / \mathrm{ml}$.

Os extratos hexânico e hidroetanolico da folha da espécie Caryocar coriaceum (pequizeiro) inibiu o crescimento radicular da $A$. cepa na concentração de $50 \mu \mathrm{g} / \mathrm{ml}$ (Cardoso et al., 2021).

Barbosa et al. (2019) avaliaram a toxicidade da fração e subfrações de diclorometano (DCM) obtidas das folhas de Smilax brasiliensis (japecanga) e observaram o efeito tóxico contra a Lactuca sativa e Allium cepa.

\section{Conclusão}

Verificou-se que os extratos da folha, casca e flor da P. platycephala não apresentaram toxicidade nas concentrações inferiores a $750 \mu \mathrm{g} / \mathrm{ml}$, entretanto na semente, esse efeito foi detectado a partir da concentração de $250 \mu \mathrm{g} / \mathrm{ml}$.

As metodologias de extração polar e sequencial, apresentaram influência direta no crescimento radicular da A. cepa. Alguns extratos apresentaram inibição do crescimento radicular, ou seja, são extratos alelopáticos, sendo portando potenciais alvos de estudos futuros, como pesticidas de origem natural.

Sugere-se realizar testes in vivo para a determinação da citotoxicidade e da toxicidade aguda dos extratos, dando assim prosseguimento aos estudos sobre a $P$. platycephala com fins de ratificar o seu uso seguro como indicação terapêutica.

\section{Referências}

Almeida, L. M., Prado, A. D. L., Xavier-Silva, K. R., Firmino, M. T., Paula, M. I. M, Gomes, P. N., Paula, J. A. M. \& Bailão, E. F. L. C. (2021). Cytotoxic effect of Vernonanthura polyanthes leaves aqueous extracts. Brazilian Journal of Biology, 81(3),575-583. https://doi.org/10.1590/1519-6984.225281

Agência Nacional de Vigilância Sanitária - ANVISA. (2011). Fundação Oswaldo Cruz (RJ). Farmacopeia Homeopática Brasileira (3a ed). Brasília: ANVISA. Disponível em: http://antigo.anvisa.gov.br/en_US/farmacopeia-homeopatica. Acesso em 28/01/2022.

Bawa-Allah, K. A. \& Akinnuoye, B. O. (2019). Acute toxicity and adverse effects of aqueous and ethanol extracts of Parkia biglobosa pods on biochemical parameters of Clarias gariepinus. African Journal of Aquatic Science, 44(1), 89-95. https://doi.org/10.2989/16085914.2018.1559794

Bagatini, M. D., Silva, A. C. F. \& Tedesco, S. B. (2007). Uso do sistema teste de Allium cepa como bioindicador de genotoxicidade de infusões de plantas medicinais. Revista Brasileira de Farmacognosia (Brazilian Journal of Pharmacognosy), 17(3),444-7. https://doi.org/10.1590/S0102-695X2007000300019

Barbosa, M. A. Fonseca, J. C., Ferraz, V., Castro, A. H. F. \& Lima, R. A. R. S. (2019). Phytotoxic and antioxidant effects of dichloromethane fraction of Smilax Brasiliensis Sprengel. Natural Product Research, 35(10),1676-1681. https://doi.org/10.1080/14786419.2019.1624955

Bonciu, E., Firbas, P., Fontanetti, C. S., Wusheng, J., Karaismailoğlu, M. C., Liu, D., Menicucci, F., Pesnya, D. S., Popescu, A., Romanovsky, A. V., Schiff, S., Ślusarczyk, J., Souza, C. P., Srivastava, A., Sutan, A. \& Papini, A. (2018). An evaluation for the standardization of the Allium cepa test as cytotoxicity and genotoxicity assay, Caryologia, 71(3), 191-209. https://doi.org/10.1080/00087114.2018.1503496

Brigido, C. F. C., Paz, M. F. C. J., Alencar, M. V. O. B., Gomes-Júnior, A. L. Ferraz, A. B. F., Grivicich, I., Cavalcante, A. A. C. M. \& Picada, J. N. (2016). Toxic effects of aqueous extract of Plectranthus amboinicus (Lour) Spreng on Allium cepa. British Journal of Pharmaceutical Research, 10(3), 1-6. https://doi.org/10.9734/BJPR/2016/23990

Cardoso, F. M., Fernandes, R. M. N., Rodrigues, M. A. M., Panontin, J. F., Scapin, E. (2021). Bioprospecção fitoquímica, atividade antioxidante in vitro e toxicidade da folha do Caryocar coriaceum do cerrado tocantinense. Gaia Scentia, 15(3):89-104. https://doi.org/10.22478/ufpb.1981-1268.2021v15n3.60156

Chiavegatto, R. B., Chaves, A. L. A., Silva, I. C. A., Lima, L. A. R. dos S., \& Techio, V. H. (2017). Cytotoxic and genotoxic effects of Solanum lycocarpum St.-Hil (Solanaceae) on the cell cycle of Lactuca sativa and Allium cepa. Acta Scientiarum. Biological Sciences, 39(2): 201-210. https://doi.org/10.4025/actascibiolsci.v39i2.34082

Fernandes, R. M. N., Rodrigues, M. A. M., Panontin, J. F., Alves, D. R., Morais, S. M., Soares, I. M. \& Scapin, E. (2021). Chemical investigation, toxic potential and acetylcholinesterase inhibitory effect of Parkia platycephala leaf and seed extracts. Journal of Medicinal Plants Research, 15(9), 401-412. https://doi.org/10.5897/JMPR2021.7158 
Freires, I. A., Sardi, J. C., Castro, R. D. \& Rosalen, P. L. (2017). Alternative animal and non-animal models for drug discovery and development: bonus or burden? Pharmaceutical Research, 34(4), 681-686. http://dx.doi.org/10.1007/s11095-016-2069-z

Inderjit, Wardle, D. A., Karban, R. . \& Callaway, R. M. (2011). The ecosystem and evolutionary contexts of allelopathy. Trends in Ecology and Evolution, 26(12), 655-662. https://doi.org/10.1016/j.tree.2011.08.00

Itta, C. Z., Magani, E. I. \& Ahom, R. I. (2014). Effectiveness of Parkia (Parkia biglobosa) products for the control of Striga genesrioides in the southern Guinea savannah. Journal of Biodiversity and. Environmental Sciences, 5(3), 36-51.

Lado, A., Sani,F. U., Yahaya,S. U. \& Karaye. A. K. (2018). Efficacy of Parkia biglobosa fruit powder on the control of Striga in cowpea cropping systems in the SudanSavanna, Nigeria. Heliyon, 4, e00733.

Magani, E. I., Ibrahim, A. \& Ahom, R. I. (2010). Sustainable control of Striga hermonthica in maize (Zea mays L.) by the use of Parkia biglobosa based products and post-emergence Herbicides. Advances in Environmental Biology 4(2), 258-264.

Mamudu, A. Y., Baiyeri, K. P. \& Echezona, B. C. (2019). Integrated weed management systems in sorghum based cropping system in Nigeria Journal of Agricultural Biotechnology and Sustainable Development, 11 (3), 20-26.

Martins, R. T. M. C., Borges, A. K. P., Armiato, A. M. \& Pimenta, R. S. (2016). Antimicrobial and phytotoxicity activities of aqueous crude extract from the Amazonian ethnomedicinal plant Bellucia grossularioides (L.) Triana. Journal of Medicinal Plants Research. 10(10), 130-138. https://doi.org/10.5897/JMPR2015.6007

Mecina, G. F., Santos. V. H. M., Dokkedal, A. L., Saldanha, L. L., Silva, L. P. \& Silva, R. M. G. (2014). Phytotoxicity of extracts and fractions of Ouratea spectabilis (Mart. ex Engl.) Engl. (Ochnaceae). South African Journal of Botany, 95, 174-180. https://doi.org/10.1016/j.sajb.2014.10.002

Oliveira, V. B., Zuchetto, M., Oliveira, C. F., Paula, C. S., Duarte, A. F. S., Miguel, M. D., Miguel, O. G. (2016). Efeito de diferentes técnicas extrativas no rendimento, atividade antioxidante, doseamentos totais e no perfil por clae-dad de Dicksonia sellowiana (presl.). Hook, dicksoniaceae. Revista Brasileira de Plantas Medicinais, 18(1), supl. I:230-239.

Panontin, J. F., Neres, R. P., Fernandes, R. M. N., Scapin, E. \& Seibert, C. S. (2021). Chemical characterization and toxicological analyses of hydroalcoholic extracts from the stem and leaves of mangabeira (Hancornia speciosa Gomes) as a guide for the development of green cosmetics. Journal of Medicinal Plants Research, 15(8): 366-379. https://doi.org/10.5897/JMPR2021.7099

Santos, K. B. D., Almeida, V. O. D., Weiler, J., \& Schneider, I. A. H. (2020). Removal of pollutants from an amd from a coal mine by neutralization/precipitation followed by "in vivo" biosorption step with the microalgae Scenedesmus sp. Minerals, 10, 711. https://doi.org/10.3390/min10080711

Soares, . IM., Rireiro, M. F., Costa, O. J., Sousa, E. E., Aguiar, A. A., Barbosa, R. S., Alvim, T. C., Ascêncio, S. D., Aguiar, R. W. S. (2017). Application of a degreasing process and sequential ultrasound-assisted extraction to obtain phenolic compounds and elucidate of the potential antioxidant of Siparuna guianensis Aublet. Journal of Medicinal Plant Research, 11(1):357-66.

Tuttolomondo, T., Licata, M., Leto, C., Bonsangue, G., Gargano, M. L., Venturella, G. \& La Bella S. (2014). Popular uses of wild plant species for medicinal purposes in the Nebrodi Regional Park (North-Eastern Sicily, Italy). Journal of Ethnopharmacoly, 157: 21-37. https://doi.org/10.1016/j.jep.2014.08.039

Viega, B. L., Rocha, A. M. \& Düsman, E. (2019). Cosmetics with hormonal composition for bioindicators Artemia salina L. and Allium cepa L. toxic potential. Environmental Science and Pollution Research 27(6), 6659-6666. https://doi.org/10.1007/s11356-019-07392-0

Young, B. J., Riera, N. I., Beily, M. E., Bres, P. A., Crespo, D. C. \& Ronco, A. E. (2012). Toxicity of the effluent from an anaerobic bioreactor treating cereal residues on Lactuca sativa. Ecotoxicology and Environmental Safety, 76,182-186. https://doi.org/10.1016/j.ecoenv.2011.09.019

Zafred, R. R. T., Spano, M. A., Martins, G. R., Figueiredo, C. C. M., Ferreira, P. C., Silva, L. P. \& Silva, R. M. G. (2016). Pro-oxidant activity and genotoxicity of the astronium fraxinifolium using wing smart and Allium cepa Test. Research Journal of Medicinal Plants, 10(4), 276-285. 